\section{Power and particles}

\section{String theories dominate for good reason.}

F or decades, high-energy physicists have basked in the glow of a theory that can apparently do no wrong. Prosaic though the 'standard model' may sound, every experimental observation from accelerators has fulfilled its predictions, often to extraordinary precision. It is also falsifiable, in that it predicts particles that should be observed in the new experimental regimes to be explored by the Large Hadron Collider at CERN from 2007 or 2008 onward. And it's conceptually sweet, exploiting mathematical symmetries to integrate three of the fundamental forces - electromagnetism and the strong and weak nuclear forces - within a single framework.

It has its weaknesses and embarrassments, however. It doesn't predict particle masses: these and many other observed parameters have to be plugged in by hand. Also, it has infinities, due to the fact that its fundamental particles are point-like. A technique ('renormalization') that gets around these infinities was a great piece of inventiveness and enables the model to deliver its sweeping successes, yet also seems like a sleight-of-hand.

Now imagine a theory that incorporates the standard model within it, that includes just one arbitrary parameter rather than many, incorporates the fourth fundamental force - gravity - within its framework, removes the need for renormalization, allows us to describe the extreme conditions of the earliest moments of the Big Bang and also resolves long-frustrating mismatches between quantum mechanics and relativity. Imagine also that such a model requires new types of mathematics in order to make progress, and that work on it repeatedly reveals levels of order that had not previously been appreciated or even suspected.

There is no theory exactly like the above, but string theory is the closest we have to it. The fundamental entities are no longer pointlike but have a finite extent. The vibrations of these features of spacetime should in principle give rise to observed particles. Even the current embryonic theories suggest observable characteristics - for example, the liquid rather than gaseous nature of the quark-gluon 'porridge' produced in collisions of gold nuclei. They also suggest enticing insights into controversies surrounding the thermodynamics of black holes.

These are no more than suggestions, and major new uncertainties have opened up alongside new opportunities. There seems to be a vast number of possible universes in such theories. Such embarrassments could prove fatal. But pursuing them is just as likely to lead to insights that make the theory seem all the more inevitable.

Two recent books have attacked the dominance of string theory among the high-energy theoretical community (see pages 491 and 507). They allege that string theory should have proved itself by now and should have made testable predictions. They also claim that string theorists have exerted an intellectual hegemony, accompanied by downright arrogance, over the field that has discouraged alternatives from being pursued.

The complaints of undue influence and arrogance are not without foundation. And string theory is very far from experiment. There is no point in making predictions prematurely, but some theorists seem to positively revel in the lack of them. A prime goal must be to turn this project, ultimately, into a testable science.

This remoteness from experiment reflects the magnitude of the task. It doesn't justify any suggestion that string theory is played out. The many theorists excited by string theory (perhaps ten times as many as those excited by any competing idea, to judge by the meetings) are going where they think the most innovative and intriguing prospects are to be found.

What drives them? There may be esoteric beauties perceivable to some string cognoscenti. But much more compelling is the sheer scope of what string theories seem to encompass. Such power, as expressed in the community, has attracted resentment and embitterment. But this power offers insight of unmatched depth and breadth, and the development of its mathematical foundations has been full of tantalizing incident.

Critical-mindedness is integral to all scientific endeavour, but the pursuit of string power deserves undaunted encouragement.

\title{
One small step
}

\section{Nature Nanotechnology will spearhead rapid progress in understanding the nanoscale.}

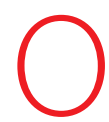

ver the past 20 years or so, Nature has published several landmark papers in nanotechnology, including the discoveries of $\mathrm{C}_{60}$, also known as buckyballs (H. W. Kroto et al. Nature $318,162-163 ; 1985)$, and of carbon nanotubes (S. Iijima Nature $354,56-58$; 1991). But the sheer volume of papers published in this sphere is rapidly expanding, and the flow of exciting science already far exceeds our capacity to publish it in this general scientific journal. Hence this month's launch of Nature Nanotechnology.

The papers in its first issue reflect the breadth of the field, with contributions as diverse as the first demonstration of a carbon-nanotube superconducting quantum-interference device and the development of a memory device based on a virus. The multidisciplinary nature of this work is, of course, one of the hallmarks of the discipline.

Research agencies and industrial companies around the world are now investing heavily in nanotechnology, in anticipation not just of scientific results but also of a substantial economic return. In electronics, for example, a big reduction in the scale of semiconductor circuitry would permit techniques that operate only at the nanoscale, such as molecular computing. Other applications are expected in manufacturing, materials, energy and environmental technology. There are also tremendous opportunities in medicine - particularly in imaging and drug delivery.

Past experience suggests that the launch of this new research journal will strengthen, rather than weaken, Nature itself, which will continue to publish papers in this field. Nature Nanotechnology will benefit authors and readers by providing greater exposure for the topic, as well as healthy competition for established journals. We are confident that it will play a central role in the probing of this valuable sphere of knowledge. 